\title{
High Absorption and Second-Harmonic Generation in Split Ring Resonator Multilayer Nanostructure
}

\author{
Renlong Zhou, Mengxiong Wu, Hui Deng, Qiong Liu, Suxia Xie, Lingxi Wu, \\ Guozheng Nie, and Jie Zhan \\ School of Physics and Electronic Science, Hunan University of Science and Technology, Xiangtan 411201, China \\ Correspondence should be addressed to Renlong Zhou; rlzhou@mail.sitp.ac.cn
}

Received 28 April 2014; Accepted 23 June 2014; Published 13 July 2014

Academic Editor: Renyun Zhang

Copyright (c) 2014 Renlong Zhou et al. This is an open access article distributed under the Creative Commons Attribution License, which permits unrestricted use, distribution, and reproduction in any medium, provided the original work is properly cited.

\begin{abstract}
Second-harmonic generation in split ring resonator multilayer nanostructure is studied with the finite-difference time-domain (FDTD) method. The fundamental frequency wave and the second-harmonic generation at the resonant absorption wavelength are highly localized in the dielectric layer, and the absorption peak is sensitive to dielectric constant of the dielectric layer. Under the excitation of the plasmon resonances mode, the strong local field induces an expected increase of the second-harmonic generation with conversion efficiencies $10^{-6}-10^{-7}$. The distributions of fundamental frequency electric field and second-harmonic electric field inside the central dielectric layer region are also shown.
\end{abstract}

\section{Introduction}

In the area of photonics, two types of materials are being developed: photonic band gap materials and metamaterials [1-8]. Split ring resonator nanostructure is the artificially structured materials that simultaneously possess plasmonic resonance features and low symmetry [9-15]. The first property provides the extraordinary linear optical electromagnetic (EM) properties. The metamaterials nanostructure with controllable permittivity and permeability can exhibit enhanced transmission of incident light involving surface plasmon polaritons (SPPs) and promises many potential applications. The second is a necessary condition for efficient second-harmonic generation (SHG). The large local field induced by the fundamental light plays an important role in the SHG processes. The properties of enhancing SHG in metallic nanostructures with low symmetry have been discussed widely, such as those in single nanoparticle and double plasmonic resonance [16, 17]. Feth et al. experimentally observed strong SHG from planar noncentrosymmetric structures with a variety of unit cell geometries [18]. The results of their experiments are interpreted successfully by a classical theory $[19,20]$. When the fundamental frequency wave (FFW) and the second harmonic wave (SHW) are both tuned at the resonant modes or defect states, giant enhancement of SHG will be obtained. Perfect absorbers for refractive index sensing and subsampling infrared imaging were also achieved by three-dimensional electromagnetic metamaterials [21-26].

In this paper, all-optical absorption is realized for the FFW and the SHW in a three-dimensional split ring resonator multilayer nanostructure by introducing resonant cavities to achieve better local field enhancement and perfect absorption. The structure consists of a split ring resonator, a dielectric layer, and a metallic layer on a glass substrate for allowing near-perfect nanostructure absorption. The proposed structure is numerically investigated by the FDTD method. The electromagnetic fields of FFW and SHW are highly localized in the dielectric layer, and the absorption peak is sensitive to dielectric constant of the dielectric layer. Under the excitation of the plasmon resonances modes, the strong local field induces an expected increase of the secondharmonic generation with conversion efficiencies of $10^{-6}$. $10^{-7}$. This novel characteristic can find important applications in all-optical integrated photonic circuits for thermal detection, imaging, solar cells, and sensing.

\section{Structure and Principles}

The numerical calculations presented in this paper have been performed with FDTD method. The method has been described elsewhere [18] and used for the analysis of 
both the linear response and the second-order nonlinearity response of plasmonic nanostructures $[19,20]$. Both the linear response and the second-order nonlinearity response of the split ring resonator multilayer nanostructure can be considered as follows:

$$
\begin{gathered}
\frac{\partial \mathbf{B}^{(1,2)}}{\partial t}=-\nabla \times \mathbf{E}^{(1,2)}, \quad \frac{\partial \mathbf{E}^{(1,2)}}{\partial t}=c^{2} \nabla \times \mathbf{B}^{(1,2)}-\frac{1}{\varepsilon_{0}} \mathbf{j}^{(1,2)}, \\
\frac{\partial \mathbf{j}^{(1)}}{\partial t}=-\gamma \mathbf{j}^{(1)}+\frac{e^{2} n_{0}}{m_{e}} \mathbf{E}^{(1)}, \\
\frac{\partial \mathbf{j}^{(2)}}{\partial t}=-\gamma \mathbf{j}^{(2)}+\frac{e^{2} n_{0}}{m_{e}} \mathbf{E}^{(2)}+\mathbf{S}^{(2)}, \\
\mathbf{S}^{(2)}=\sum_{k} \frac{\partial}{\partial r_{k}}\left(\frac{\mathbf{j}^{(1)} j^{(1)}}{e n_{0}}\right) \\
-\frac{e}{m_{e}}\left[\varepsilon_{0}\left(\nabla \cdot \mathbf{E}^{(1)}\right) \mathbf{E}^{(1)}+\mathbf{j}^{(1)} \times \mathbf{B}^{(1)}\right] \quad(k=x, y, z),
\end{gathered}
$$

where $\mathbf{S}^{(2)}$ is the nonlinear source for second-order nonlinearity. $\mathbf{E}^{(1)}$ and $\mathbf{E}^{(2)}, \mathbf{B}^{(1)}$ and $\mathbf{B}^{(2)}$, and $\mathbf{J}^{(1)}$ and $\mathbf{J}^{(2)}$ are the electric field, the magnetic flux intensity vectors, and the current density vectors of fundamental and harmonic waves, respectively. $n_{0}$ is the ion density, $m_{e}$ is the electron mass, $c$ the light speed in vacuum air, and $e$ is the elementary charge.

The permittivity of gold has the following form: $\varepsilon_{r}(\omega)=$ $1-\omega_{p}^{2} / \omega(\omega+i \gamma)$. The $\gamma=6.478 \times 10^{13} \mathrm{~s}^{-1}$ is phenomenological collision frequency, and $\omega_{p}=\sqrt{e^{2} n_{0} / m_{e} \varepsilon_{0}}=1.367 \times 10^{16} \mathrm{~s}^{-1}$ is the bulk plasma frequency.

The split ring resonator multilayer nanostructure under study is schematically shown in Figure 1, where the threedimensional unit cell view is depicted. It consists of split ring resonator and gold layer separated by a dielectric layer and a glass substrate under the lower gold layer. Two-dimensional split ring resonator arrays are deposited on the top dielectric layer with the same period in $x$ and $y$ directions. The perfectly matched absorbing boundary conditions are employed at the bottom and the top of the computational space along the $z$ direction, and the periodic boundary conditions are used on the boundaries of $x$ and $y$ directions. Only one unit cell of the periodic holes array is considered in our computational space. To investigate the absorption properties of the proposed multilayer nanostructure, numerical simulations were performed by the three-dimensional FDTD method.

The multilayer nanostructures have periodic modulation on both the linear and the second-order susceptibility. The lattice periodic $a_{x}$ and $a_{y}$ are along the $x$ and $y$ directions, respectively. To investigate the influences of the structure parameters on the proposed metamaterial absorption, first we use the parameters with the split ring resonator depth $t_{1}=70 \mathrm{~nm}$, dielectric depth $t_{2}=70 \mathrm{~nm}$, below gold layer depth $t_{3}=210 \mathrm{~nm}$, below glass layer depth $t_{4}=280 \mathrm{~nm}$, and structure periods $a_{x}$ and $a_{y}$ equal to $a=700 \mathrm{~nm}$. There is the unit cell of split ring resonator shape with $b=504 \mathrm{~nm}$,

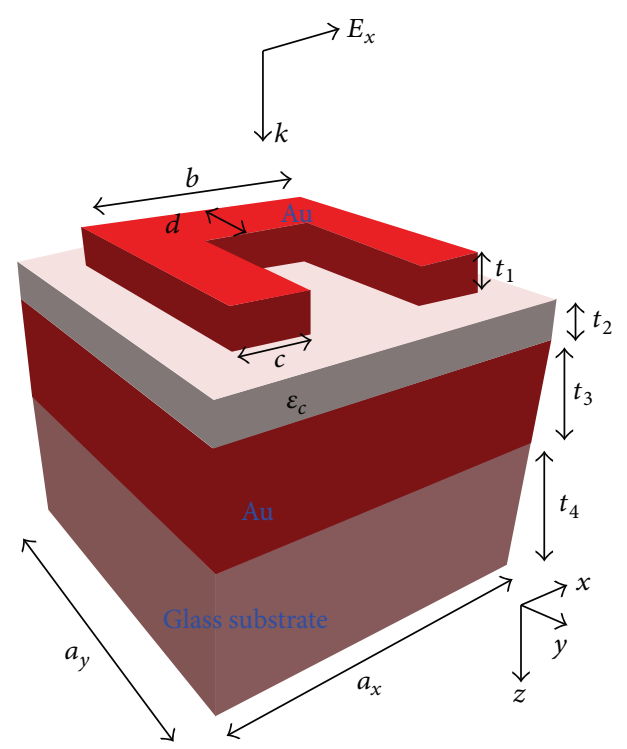

FIGURE 1: The split ring resonator multilayer nanostructure with the lattice periodic $a_{x}$ and $a_{y}$ along the $x$ and $y$ directions, respectively. The input light wave is polarized along the $x$ direction and propagates along the $z$ direction.

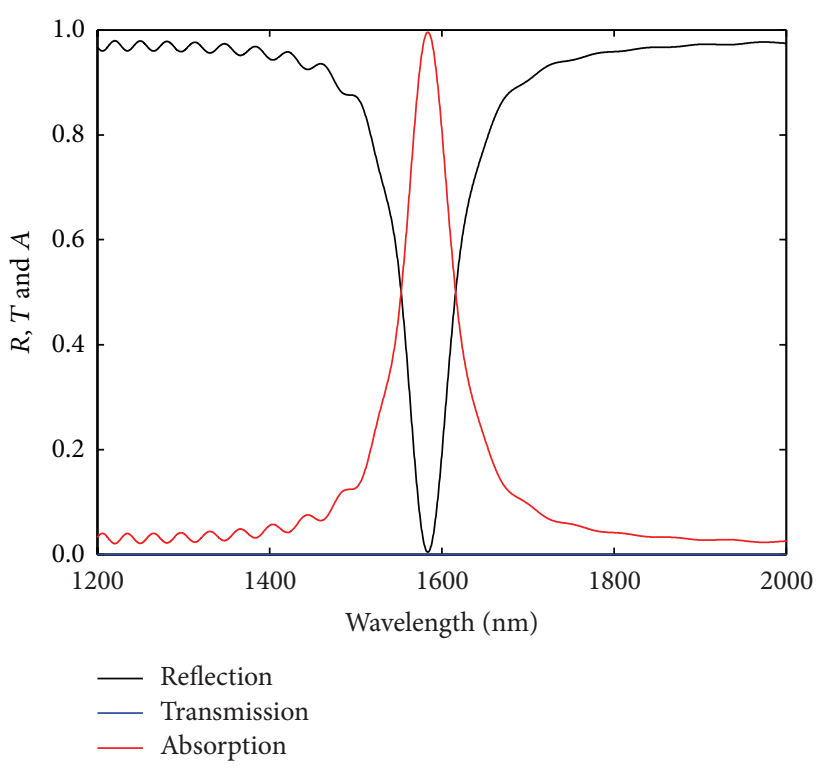

FIGURE 2: Spectral properties of the proposed split ring resonator multilayer nanostructure: reflection $(R)$, transmission $(T)$, and absorption (A).

$c=140 \mathrm{~nm}$, and $d=70 \mathrm{~nm}$. In the calculation, the incident light wave with polarization along $x$ direction is normally illuminated to the split ring resonator multilayer nanostructure.

\section{Simulation Results and Discussions}

The reflection $(R)$, transmission $(T)$, and absorption $(A)$ are calculated and demonstrated in Figure 2. Structure absorption is defined by $A=1-R-T$, thus the key to obtain 

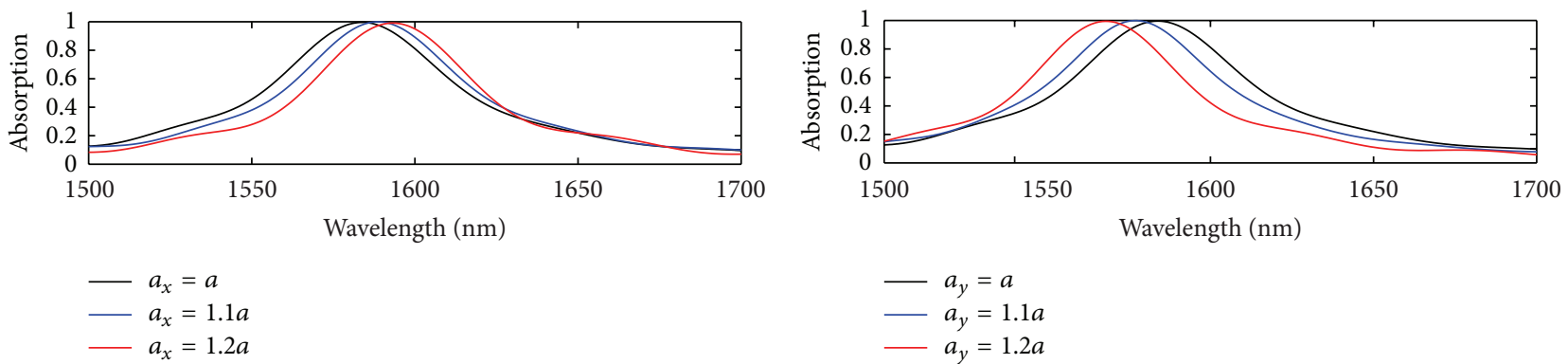

$$
\begin{aligned}
-a_{y} & =a \\
-a_{y} & =1.1 a \\
-a_{y} & =1.2 a
\end{aligned}
$$

(a)

(b)

Figure 3: Absorption of FFW for lattice constant (a) along $x$ direction $a_{x}=a$ (black line), 1.1 $a$ (blue line), and 1.2a (red line) and (b) along $y$ direction $a_{y}=a$ (black line), $1.1 a$ (blue line), and $1.2 a$ (red line), respectively.

high absorption is to simultaneously minimize $R$ and $T$. From Figure 2, it can be clearly obtained that $T$ is zero at all the frequencies. This is because the depth of gold film on the glass substrate is larger than skin depth of light, and the incident light cannot penetrate gold film. In the reflection spectrum, a significant resonant dip occurs at wavelength of $1584 \mathrm{~nm}$, which results in a narrow-band peak as high as 1.0 in the absorption spectrum. The electromagnetic resonance response can be tuned by the structure parameters, and it provides the possibility to match the impedance $Z=(\mu / \varepsilon)^{0.5}$ to free space at a specific frequency [21]. Therefore, when the wave at this frequency propagates to the three-dimensional split ring resonator multilayer nanostructure, no energy will be reflected. As a result, a resonant dip occurs in the reflection spectrum. In this case, the incident optical energy is neither transmitted nor reflected, thereby leading to nearly $100 \%$ absorbance of the proposed structure.

Now, the dependences of the absorption spectra on the split ring resonator multilayer nanostructure parameters are investigated. When enlarging the structure period $a_{x}$ along $x$ direction or the structure period $a_{y}$ along $y$ direction from $700 \mathrm{~nm}$ to $770 \mathrm{~nm}$ and $840 \mathrm{~nm}$, while maintaining other structure parameters the same as the design in Figure 2, the absorption spectra of FFW through a split ring resonator multilayer nanostructure are calculated in Figures $3(\mathrm{a})$ and 3(b). It illustrates that the absorption peak can be linearly tuned by the multilayer nanostructure period along $x$ direction and along $y$ direction. When the multilayer nanostructure period along $x$ direction is increased, a red shift of absorption peak is observed. When the multilayer nanostructure period along $y$ direction is increased, a blue shift of absorption peak is observed.

The influence of the wide $d$ of split ring resonator along $y$ direction on the absorption of FFW through a split ring resonator multilayer nanostructure is shown in Figure 4, where the period is fixed at $700 \mathrm{~nm}$ and the wide $d$ of split ring resonator is changed from $d=70 \mathrm{~nm}$ to $140 \mathrm{~nm}$ and $210 \mathrm{~nm}$, respectively. When the wide $d$ of split ring resonator is $d=$ $210 \mathrm{~nm}$, a significant resonant dip occurs at the wavelength of $1517 \mathrm{~nm}$, which thereby results in a narrow-band peak as high as 0.8 in the absorption spectrum. When the wide $d$ of split ring resonator is increased, a blue shift of absorption peak is observed. It can be clearly obtained that the wide $d$

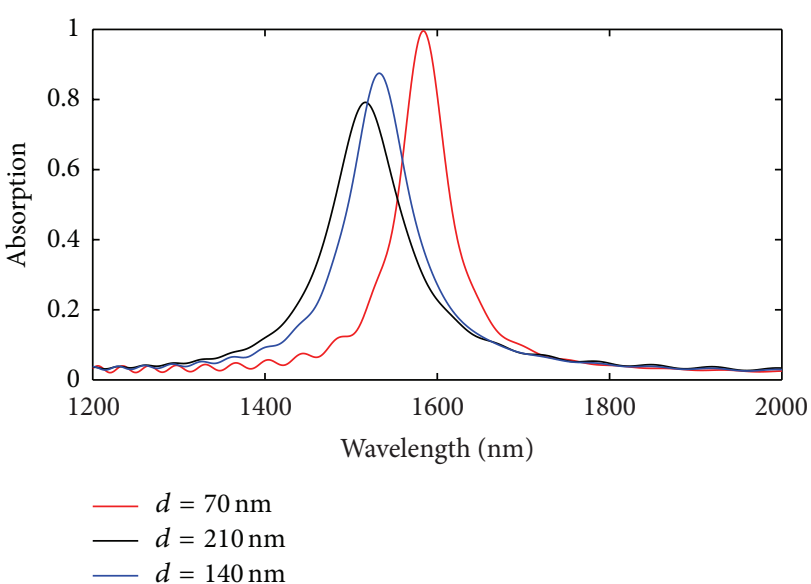

FIGURE 4: Absorption of FFW for different wide $d=70 \mathrm{~nm}$ (red line), $140 \mathrm{~nm}$ (blue line), and $210 \mathrm{~nm}$ (black line) of split ring, respectively.

of split ring resonator will strongly influence the position and intensity of absorption peak. The reason is that increasing the wide $d$ of split ring will minimize the interactions regions of upper and lower metallic layers, leading to the impedance mismatch, and thus the structure absorption phenomenon will be deteriorated.

The influence of the wide $c$ of split ring resonator arms on the absorption of fundamental frequency wave through a split ring resonator multilayer nanostructure is shown in Figure 5, where the period is fixed at $700 \mathrm{~nm}$ and the wide $c$ of split ring resonator arms is changed from $c=140 \mathrm{~nm}$ (black line) to $224 \mathrm{~nm}$ (blue line) and $238 \mathrm{~nm}$ (red line), respectively. It can be obtained that the wide $c$ of split ring resonator arms will strongly influence the position of absorption peak. The reason is that increasing the wide $c$ of split ring will minimize the interactions regions of upper and lower metallic layers and thus the structure absorption phenomenon will be deteriorated.

The influence of the dielectric constant $\varepsilon_{c}$ of the dielectric layer on the absorption of fundamental frequency wave through a split ring resonator multilayer nanostructure is shown in Figure 6(a), where the period is fixed at $700 \mathrm{~nm}$ and the dielectric constant $\varepsilon_{c}$ of the dielectric layer is changed 


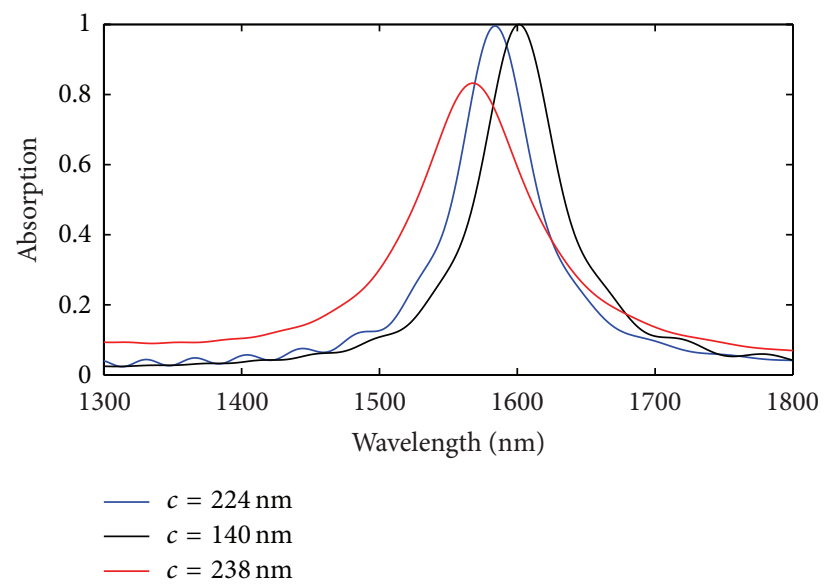

FIGURE 5: Absorption of FFW for different wide $c=140 \mathrm{~nm}$ (black line), $224 \mathrm{~nm}$ (blue line), and $238 \mathrm{~nm}$ (red line), respectively.

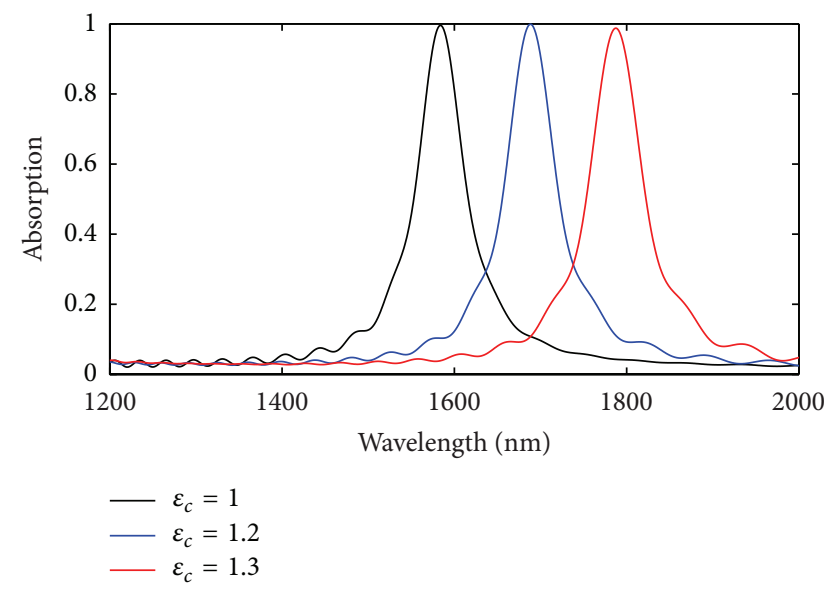

(a)

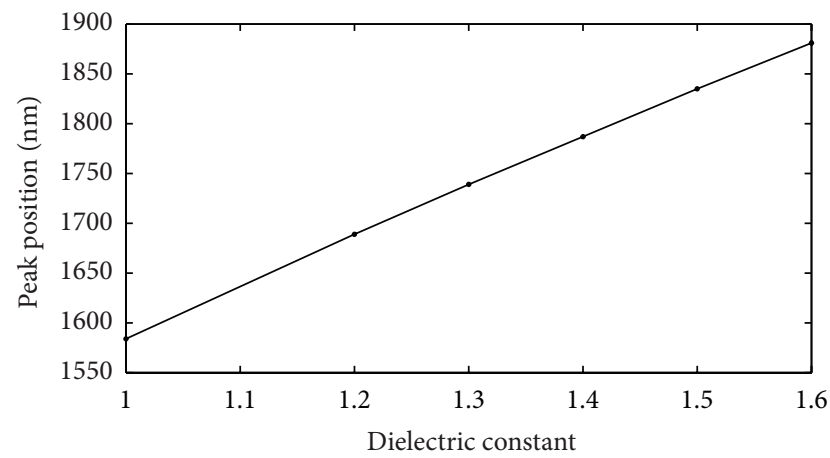

(b)

Figure 6: (a) Absorption of FFW for different dielectric constant $\varepsilon_{c}=1.0$ (black line), 1.2 (blue line), and 1.4 (red line), respectively. (b) The sensitivity of the absorption peak to the dielectric constant.

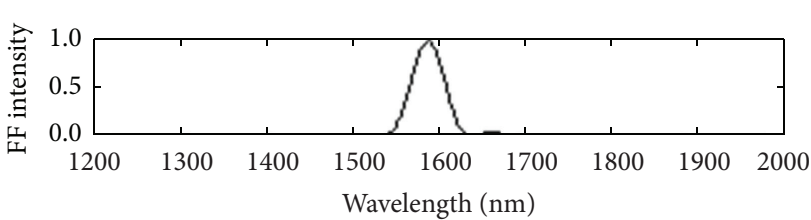

(a)

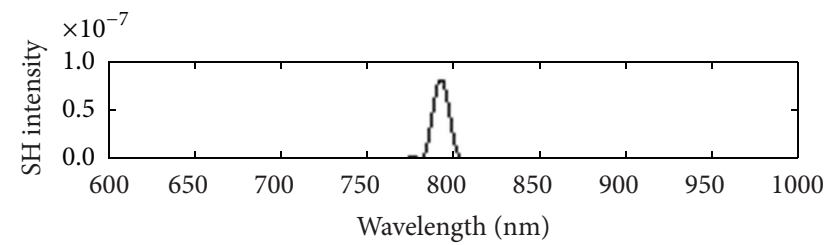

(b)

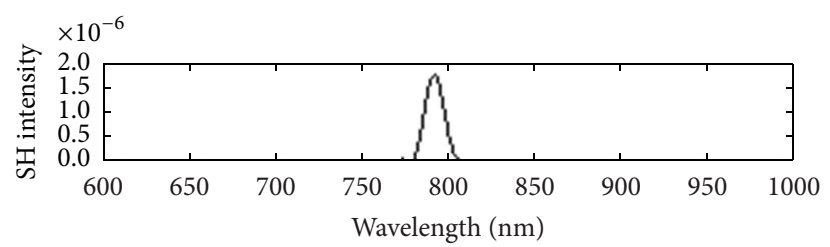

(c)

Figure 7: The absorption spectra of (a) fundamental frequency field $E_{x}^{(1)}$, (b) second-harmonic field $E_{x}^{(2)}$, and (c) second-harmonic field $E_{y}^{(2)}$ are calculated for the wide $d=210 \mathrm{~nm}$, while maintaining other structure parameters the same as the design in Figure 2. 


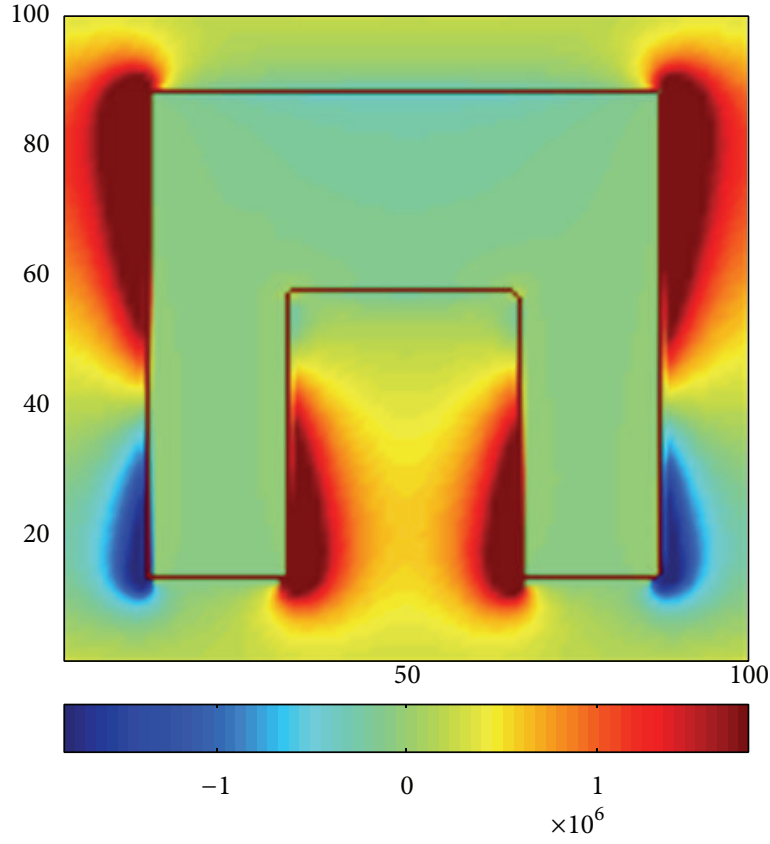

(a)

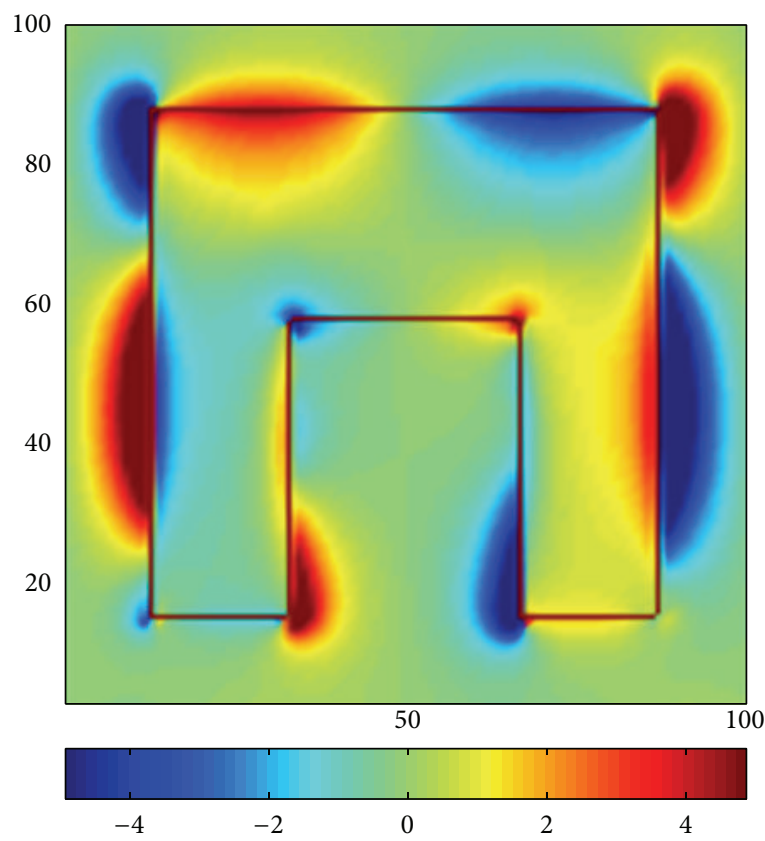

(c)

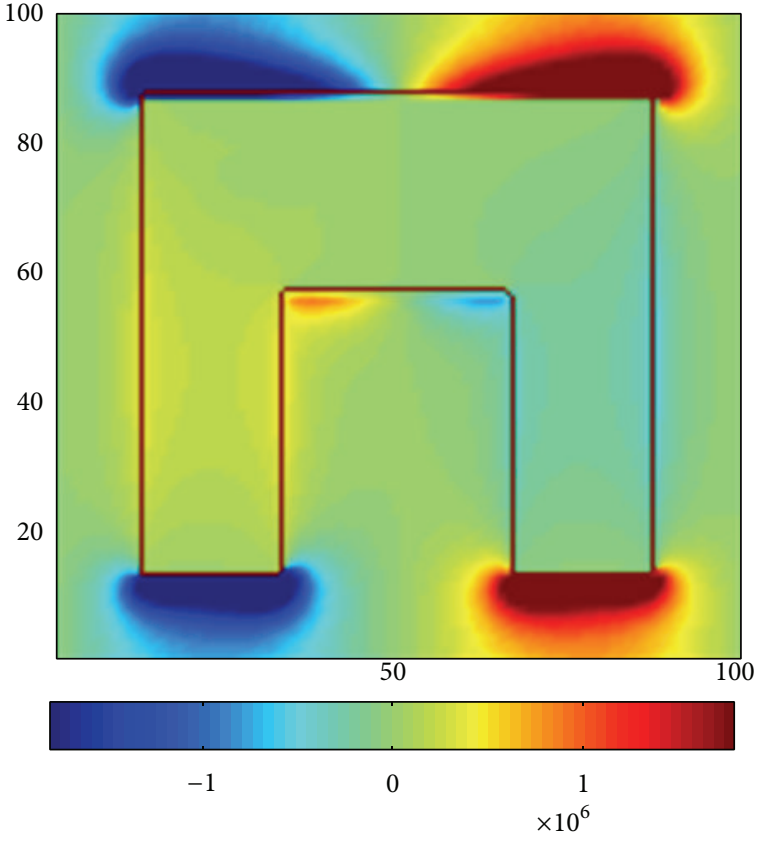

(b)

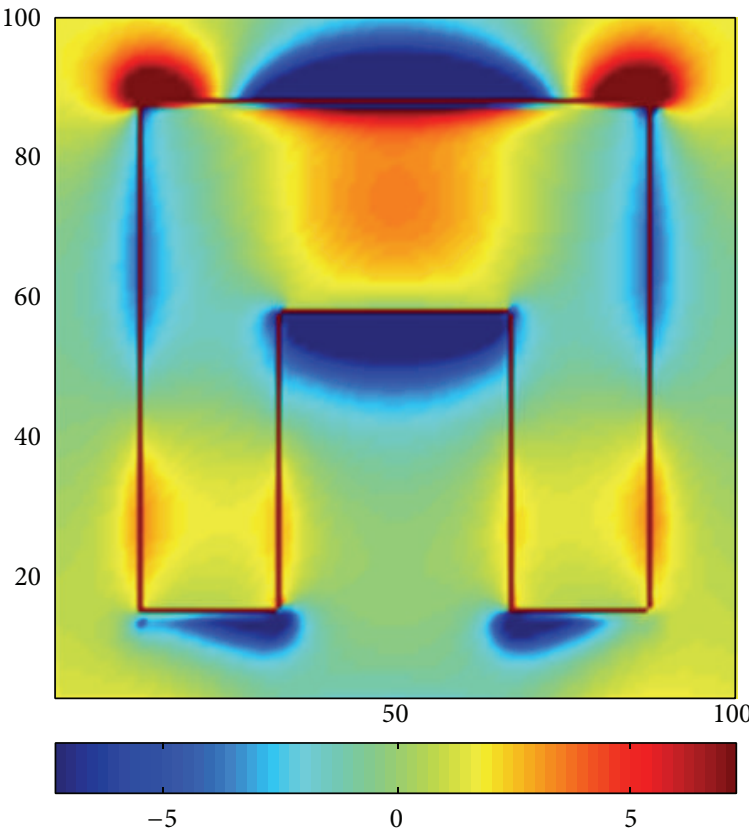

(d)

Figure 8: The distribution of fundamental frequency fields (a) $E_{x}^{(1)}$ and (b) $E_{y}^{(1)}$ inside the central dielectric layer at wavelength $1518 \mathrm{~nm}$. The distribution of second-harmonic fields (c) $E_{x}^{(2)}$ and (d) $E_{y}^{(2)}$ in the same position at wavelength $759 \mathrm{~nm}$.

from $\varepsilon_{c}=1$ (black line) to 1.2 (blue line) and 1.4 (red line), respectively. It can be obtained that the dielectric constant $\varepsilon_{c}$ of the dielectric layers will strongly influence the position of absorption peak but will not reduce the absorption peak. In Figure 6(b), sensitivity of the absorption peak to the dielectric constant $\varepsilon_{c}$ of the dielectric layer is plotted. When $\varepsilon_{c}$ is changed from 1.0 to 1.6 , the absorption peak varies almost linearly from $1583.8 \mathrm{~nm}$ to $1688.2 \mathrm{~nm}$ and
$1787.1 \mathrm{~nm}$, respectively. The results in Figures 6(a) and 6(b) indicate two properties of the proposed structure, that is, the electromagnetic field is highly localized in the dielectric layer, and the absorption peak is sensitive to dielectric constant of the dielectric layer. These two novel characteristics ensure us to actively tune the all-optical metamaterial absorption.

To obtain the absorption spectra of SHG of the threedimensional split ring resonator multilayer nanostructure, 


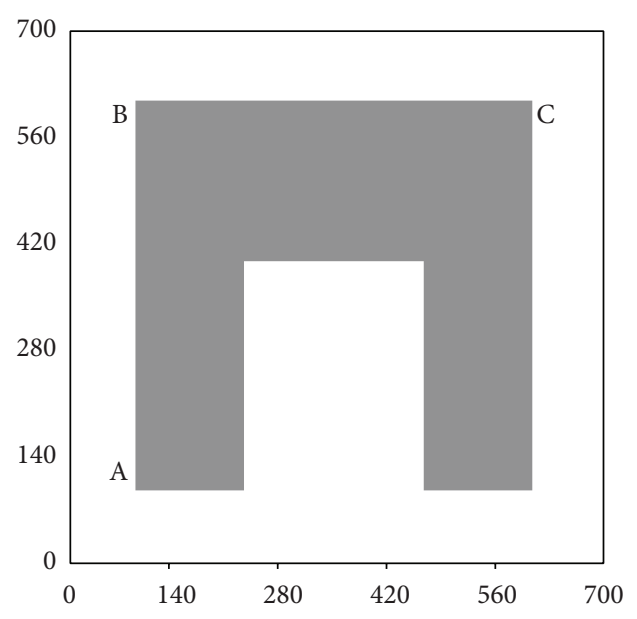

(a)

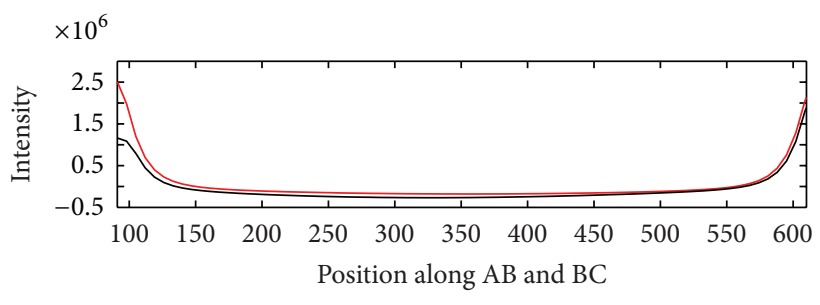

(b)

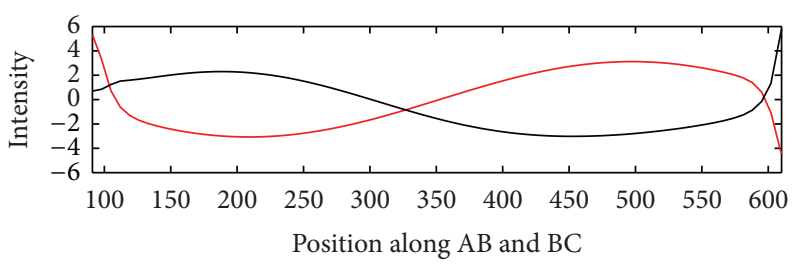

(c)

Figure 9: (a) The structure of split ring on $x y$ plane. (b) The fundamental frequency field distribution $E_{x}^{(1)}$ along the BC (red line) and $E_{y}^{(1)}$ along the $\mathrm{AB}$ (black line) at wavelength $1518 \mathrm{~nm}$. (c) The second-harmonic electric field $E_{x}^{(2)}$ along the $\mathrm{BC}$ (red line) and $E_{y}^{(2)}$ along the $\mathrm{AB}$ (black line) at wavelength $759 \mathrm{~nm}$.

the input light wave $E_{x}^{(1)}, E_{x}^{(1)}=E_{0} \sin \left(2 \pi c t / \lambda_{0}\right)$, is polarized along the $x$ direction with wavelengths $\lambda_{0}$, and the $E_{0}$ is amplitude.

The absorption spectra of fundamental frequency field $E_{x}^{(1)}$, second-harmonic field $E_{x}^{(2)}$, and second-harmonic field $E_{y}^{(2)}$ are calculated in Figures $7(\mathrm{a})$ and $7(\mathrm{c})$ for the wide $d=$ $210 \mathrm{~nm}$ of split ring along $y$ direction, while maintaining other structure parameters the same as the design in Figure 2. When the continuous wave $E_{x}^{(1)}$ at incident wavelengths $\lambda_{1}=$ $1518 \mathrm{~nm}$ is incident through the split ring resonator, one can see absorption spectra of fundamental frequency wave at the wavelength $1518 \mathrm{~nm}$ in Figure 7(a). The absorption spectra of the second-harmonic field $E_{x}^{(2)}$ and the $E_{y}^{(2)}$ component at the wavelength $759 \mathrm{~nm}$ are also shown in Figures 7 (b) and $7(\mathrm{c})$, respectively. The second harmonic conversion efficiencies in the second-order nonlinear optical process are defined as follows: $\eta=\left|E^{(2)}\left(2 \omega_{0}\right) / E^{(1)}\left(\omega_{0}\right)\right|^{2}$, where $\omega_{0}$ is the frequency of the incident FFW. The $y$-polarized second harmonic conversion efficiencies are about $10^{-6}$ while the $x$-polarized SH conversion efficiencies are about $10^{-7}$ for the $x$-polarized fundamental frequency wave incident at the wavelength $1518 \mathrm{~nm}$.

The transmission of the FFW results from an enhancement of the local field. The strong local field and noncentrosymmetry induce an increase of second harmonic nonlinearity signals. A strong electromagnetic field is established at the resonant wavelength and localized in the dielectric layer region. The electric field distributions of $E_{x}^{(1)}$ and $E_{y}^{(1)}$ for fundamental frequency field inside the central dielectric layer region at wavelength $1518 \mathrm{~nm}$ are shown in Figures 8(a) and $8(\mathrm{~b})$, respectively. And the second-harmonic electric field distributions of $E_{x}^{(2)}$ and $E_{y}^{(2)}$ for SHG inside the central dielectric layer region at wavelength $759 \mathrm{~nm}$ are also shown in Figures 8 (c) and 8(d), respectively. The distributions of fundamental frequency electric field $E_{x}^{(1)}$ along the BC (red line) and $E_{y}^{(1)}$ along the $\mathrm{AB}$ direction (black line) at wavelength $1518 \mathrm{~nm}$ are shown in Figure 9(b). The distributions of the secondharmonic electric field $E_{x}^{(2)}$ along the BC direction (red line) and $E_{y}^{(2)}$ along the $\mathrm{AB}$ (black line) at wavelength $759 \mathrm{~nm}$ are shown in Figure 9(c).

\section{Conclusions}

In summary, we have numerically investigated a threedimensional split ring resonator multilayer nanostructure. The structure consists of split ring resonator and metallic layers for allowing near-perfect absorption of the fundamental frequency wave and the second-harmonic generation. The electromagnetic field is highly localized in the dielectric layer, and the absorption peak is sensitive to dielectric constant of the dielectric layer. These characteristics ensure us to tune the metamaterial absorption. Under the excitation of the plasmon resonances modes, the strong local field induces an expected increase of the second-harmonic generation. The 
second harmonic conversion efficiency is about $10^{-6}-10^{-7}$. This highly nonlinear behavior of the absorption switching structure can find potential applications in all-optical integrated photonic circuits and networks, such as thermal detectors and imaging, solar cell, and plasmonic sensing.

\section{Conflict of Interests}

The authors declare that there is no conflict of interests regarding the publication of this paper.

\section{Acknowledgments}

This work was supported by the National Natural Science Foundation of China under Grants nos. 11247003, 51175172, and 11304094, the Open Research Fund of National Laboratory for Infrared Physics (GJKF20130027 and GJKF20130028), the Chinese Academy of Sciences, and the Scientific Research Fund of Hunan Provincial Education Department (12A045, 12C0143, and 13C323). The authors would especially like to thank Dr. Yong Zeng for the discussion of FDTD simulations.

\section{References}

[1] X. Chen, Y. Chen, J. Dai et al., "Ordered Aunanocry-stals on a substrate formed by light-induced rapid annealing," Nanoscale, vol. 6, pp. 1756-1762, 2014.

[2] N. Guo, W.-D. Hu, X.-S. Chen, L. Wang, and W. Lu, "Enhanced plasmonic resonant excitation in a grating gated field-effect transistor with supplemental gates," Optics Express, vol. 21, no. 2, pp. 1606-1614, 2013.

[3] L. Wang, W. Hu, J. Wang et al., "Plasmon resonant excitation in grating-gated AlN barrier transistors at terahertz frequency," Applied Physics Letters, vol. 100, no. 12, Article ID 123501, 2012.

[4] W. D. Hu, L. Wang, X. S. Chen et al., "Room-temperature plasmonic resonant absorption for grating-gate GaN HEMTs in far infrared terahertz domain," Optical and Quantum Electronics, vol. 45, no. 7, pp. 713-720, 2013.

[5] G. Li, X. Chen, B. Ni et al., "Fractal H-shaped plasmonic nanocavity," Nanotechnology, vol. 24, no. 20, Article ID 205702, 2013.

[6] G. Li, X. Chen, O. Li et al., "A novel plasmonic resonance sensor based on an infrared perfect absorber," Journal of Physics D: Applied Physics, vol. 45, no. 20, Article ID 205102, 2012.

[7] J. Liu, M. Brio, Y. Zeng et al., "Generalization of the FDTD algorithm for simulations of hydrodynamic nonlinear Drude model," Journal of Computational Physics, vol. 229, no. 17, pp. 5921-5932, 2010.

[8] M. Yan, J. Dai, and M. Qiu, "Lithography-free broadband visible light absorber based on a mono-layer of gold nanoparticles," Journal of Optics, vol. 16, Article ID 025002, 2014.

[9] M.-L. Ren and Z.-Y. Li, "Giant enhancement of second harmonic generation in nonlinear photonic crystals with distributed Bragg reflector mirrors," Optics Express, vol. 17, no. 17, pp. 14502-14510, 2009.

[10] M. Ren and Z. Li, "Exact iterative solution of second harmonic generation in quasi-phase-matched structures," Optics Express, vol. 18, no. 7, pp. 7288-7299, 2010.
[11] J. J. Li, Z. Y. Li, Y. Sheng, and D. Z. Zhang, "Giant enhancement of second harmonic generation in poled ferroelectric crystals," Applied Physics Letters, vol. 91, no. 2, Article ID 022903, 2007.

[12] J. Li, Z. Y. Li, and D. Z. Zhang, "Nonlinear frequency conversion in two-dimensional nonlinear photonic crystals solved by a plane-wave-based transfer-matrix method," Physical Review B, vol. 77, no. 19, Article ID 195127, 2008.

[13] B. Wang, R. Wang, R. J. Liu, X. H. Lu, J. Zhao, and Z. Li, "Origin of shape resonance in second-harmonic generation from metallic nanohole arrays," Scientific Reports, vol. 3, article 2358, 2013.

[14] S. Linden, F. B. P. Niesler, J. Förstner, Y. Grynko, T. Meier, and M. Wegener, "Collective effects in second-harmonic generation from split-ring-resonator arrays," Physical Review Letters, vol. 109, no. 1, Article ID 015502, 2012.

[15] V. K. Valev, A. V. Silhanek, N. Verellen et al., "Asymmetric optical second-harmonic generation from chiral G-shaped gold nanostructures," Physical Review Letters, vol. 104, no. 12, Article ID 127401, 2010.

[16] J. Butet, J. Duboisset, G. Bachelier et al., "Optical second harmonic generation of single metallic nanoparticles embedded in a homogeneous medium," Nano Letters, vol. 10, no. 5, pp. 1717-1721, 2010.

[17] S. Park, J. W. Hahn, and J. Y. Lee, "Doubly resonant metallic nanostructure for high conversion efficiency of second harmonic generation," Optics Express, vol. 20, no. 5, pp. 4856-4870, 2012.

[18] N. Feth, S. Linden, M. W. Klein et al., "Second-harmonic generation from complementary split-ring resonators," Optics Letters, vol. 33, no. 17, pp. 1975-1977, 2008.

[19] Y. Zeng, W. Hoyer, J. Liu, S. W. Koch, and J. V. Moloney, "Classical theory for second-harmonic generation from metallic nanoparticles," Physical Review B, vol. 79, no. 23, Article ID 235109, 2009.

[20] Y. Zeng and J. V. Moloney, "Volume electric dipole origin of second-harmonic generation from metallic membrane with noncentrosymmetric patterns," Optics Letters, vol. 34, no. 18, pp. 2844-2846, 2009.

[21] Z. G. Dong, H. Liu, M. X. Xu et al., "Plasmonically induced transparent magnetic resonance in a metallic metamaterial composed of asymmetric double bars," Optics Express, vol. 18, no. 17, pp. 18229-18234, 2010.

[22] J. Butet, G. Bachelier, I. Russier-Antoine, C. Jonin, E. Benichou, and P.-F. Brevet, "Interference between selected dipoles and octupoles in the optical second-harmonic generation from spherical gold nanoparticles," Physical Review Letters, vol. 105, no. 7, Article ID 077401, 2010.

[23] J. U. Fürst, D. V. Strekalov, D. Elser et al., "Naturally phasematched second-harmonic generation in a whispering-gallerymode resonator," Physical Review Letters, vol. 104, no. 15, Article ID 153901, 2010.

[24] Y. Gong, Z. Li, J. Fu et al., "Highly flexible all-optical metamaterial absorption switching assisted by Kerr-nonlinear effect," Optics Express, vol. 19, no. 11, pp. 10193-10198, 2011.

[25] N. Liu, M. Mesch, T. Weiss, M. Hentschel, and H. Giessen, "Infrared perfect absorber and its application as plasmonic sensor," Nano Letters, vol. 10, no. 7, pp. 2342-2348, 2010.

[26] L. Meng, D. Zhao, Z. Ruan, Q. Li, Y. Yang, and M. Qiu, "Optimized grating as an ultra-narrow band absorber or plasmonic sensor," Optics Letters, vol. 39, no. 5, pp. 1137-1140, 2014. 

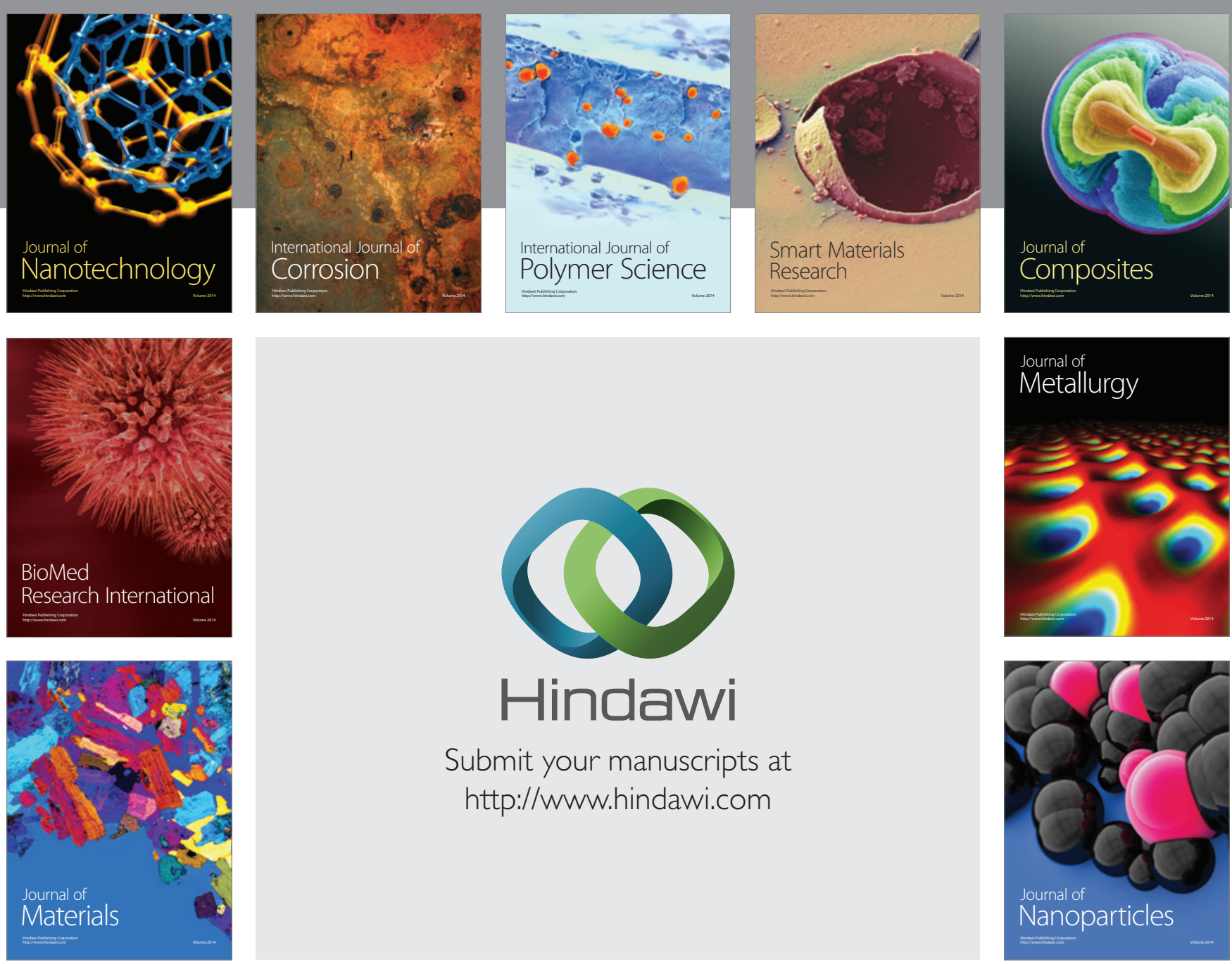

Submit your manuscripts at http://www.hindawi.com
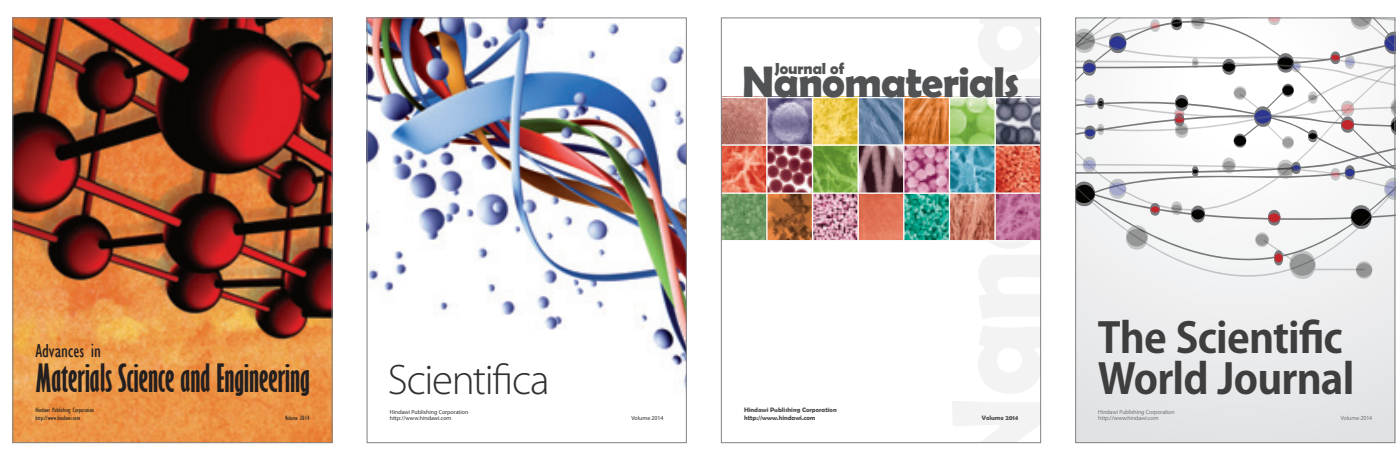

\section{The Scientific World Journal}
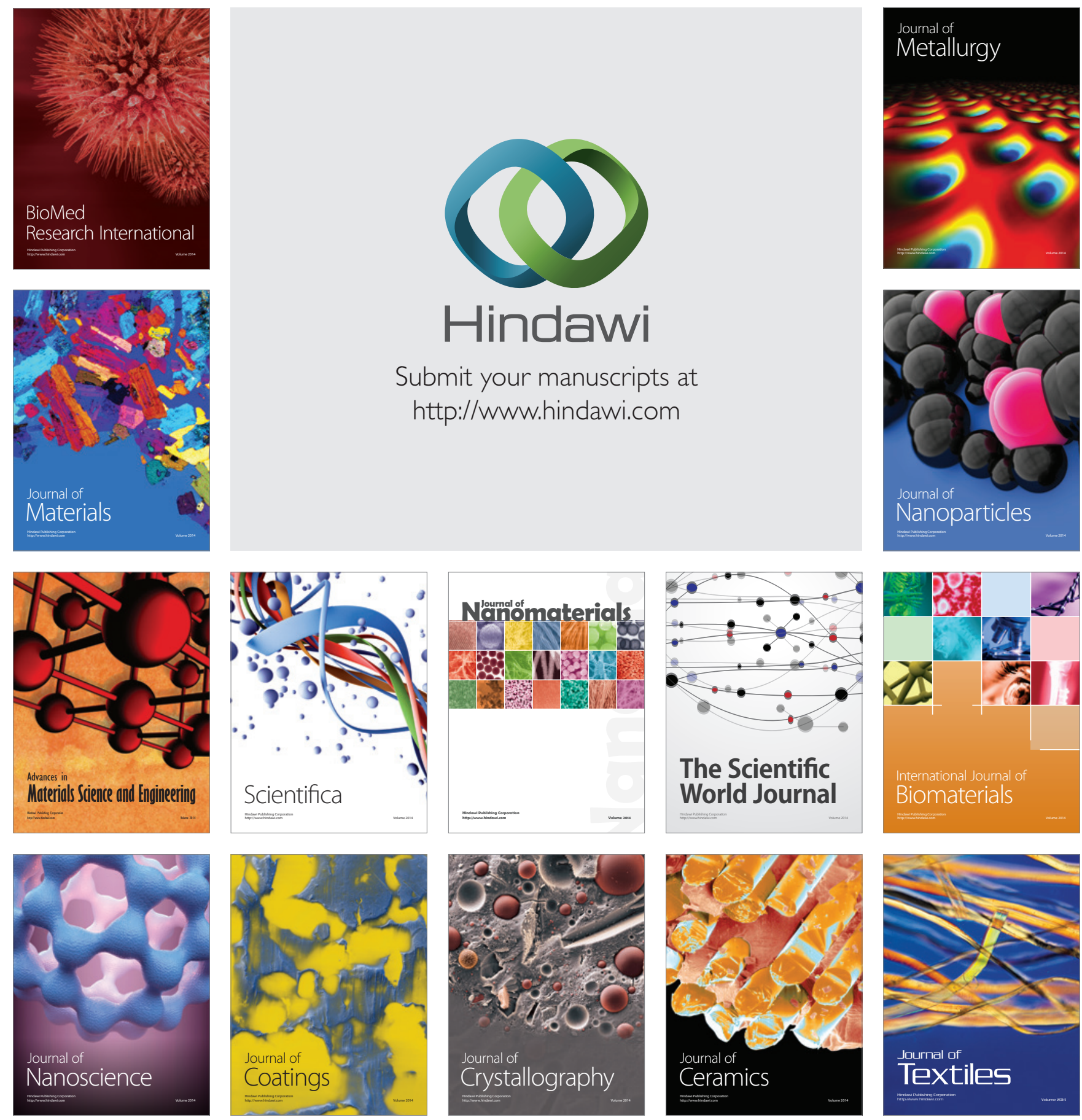